\title{
INVESTIGATING THE BEACH PREDICTIONS OF A NEW LONG-TERM NUMERICAL MORPHOLOGICAL MODEL - A CARIBBEAN CONTEXT
}

\author{
Deborah Villarroel-Lamb ${ }^{1}$
}

\begin{abstract}
A recently developed beach change model was investigated to assess its predictive capability with respect to shoreline change. This investigation formed part of a number of analyses being conducted to assess the capability of the numerical model. The model was firstly compared to a commonly used commercial model to assess its output on wave and sediment responses. Secondly, the beach changes were investigated to determine a likely probability density function for the shoreline responses. A number of probability density functions were compared with the results and critical deductions were made. Lastly, the new beach change model has a distinctive feature which attempts to reduce the model runtime to promote greater use. This wave-averaging feature was investigated to determine model performance as parameters were changed. It was shown that the model compares favorably to the commercial package in some aspects, but not all. The shoreline response may be best described by a single probability density function, which makes it quite suitable for quantitative risk analyses. Lastly, the wave-averaging feature can be used to reduce runtime although this requires the user to apply sound judgment in the analyses.
\end{abstract}

Keywords: beach morphology; numerical modeling; wave transformation modeling

\section{INTRODUCTION}

\section{Background}

All coastlines are subject to accretion and erosion processes. In addition, many areas such as the Caribbean region rely heavily on these coastlines for tourism. Tourism forms a substantial contributor to the economies of most Caribbean islands. Coastal Zone Management is a challenging task that attempts to indicate the optimal course of action in a given coastal management unit through reliance on data and data analyses. This exercise usually involves the commitment of significant resources to ensure effectiveness of the exercise and produce useful results. However, despite the dependency on their coastlines, some countries do not possess the resources to engage in actively monitoring these coasts nor to implement detailed investigations which at times require expensive tools for analyses. Most islands of the Caribbean region fall into this subset of countries that rely on the coastlines to support their economies but are unable to dedicate the resources to engage in comprehensive investigations. These countries usually need to commit the limited resources to other planning or infrastructural activities and not enough resources are allocated to coastal planning and management undertakings. Although, this trend is changing, there is still much work to be done before Caribbean countries can optimally use their coastal resources. There is still a lack of adequate data for proper planning, and a lack of state-of-the-art tools to be used in analyses and investigations. These were the limitations that precipitated the development of the coastal tool discussed in this paper. A coastal morphological numerical model was developed to be freely disseminated for use by the relevant Caribbean organizations, so that effective planning and management can occur in the coastal zones.

Certain features of the numerical tool were established to ensure that the user requires as minimal data input as possible to facilitate the easy application of the tool in a preliminary assessment. The objective is that the tool be used as an indicator of what action is required on the coastline, and will then be succeeded by more detailed analyses. To achieve this aim, a number of processes are parameterized and the user is required to calibrate and validate the output using the various parameters in the model. Another feature is the removal of the requirement to input forcing data, such as waves and currents. The model uses historical data to extract distributions and randomly samples from the relevant distributions for model forcing. The user is only required to input bathymetric data and can engage in modeling shoreline change, inter alia, driven by low to high energy waves, wave generated currents and sea-level changes. Scenario modeling for sea level changes, which at present is a critical coastal research activity, can be accomplished using the numerical model. The subsequent section describes the numerical morphological model in greater detail.

\footnotetext{
${ }^{1}$ Department of Civil and Environmental Engineering, University of the West Indies, St. Augustine, Trinidad and Tobago Deborah.Villarroel-Lamb@sta.uwi.edu
} 


\section{The Numerical Model- a detailed review}

Generally, the model couples a sediment transport module with a hydrodynamic module across both the surf and swash zones (see Figure 1). The model employs a phase-averaged, depth-integrated approach to model the relevant coastal processes, from the deep-water limit to the shoreline, and employs a finite difference scheme to solve the relevant equations. Descriptions of the morphological response parameter are derived through the random sampling of the input parameters from a defined distribution, which then provides a possible outcome of the response parameter, which is ideally the bathymetry across the surf and swash zones. The investigation of this response parameter is the focal point of this research paper.

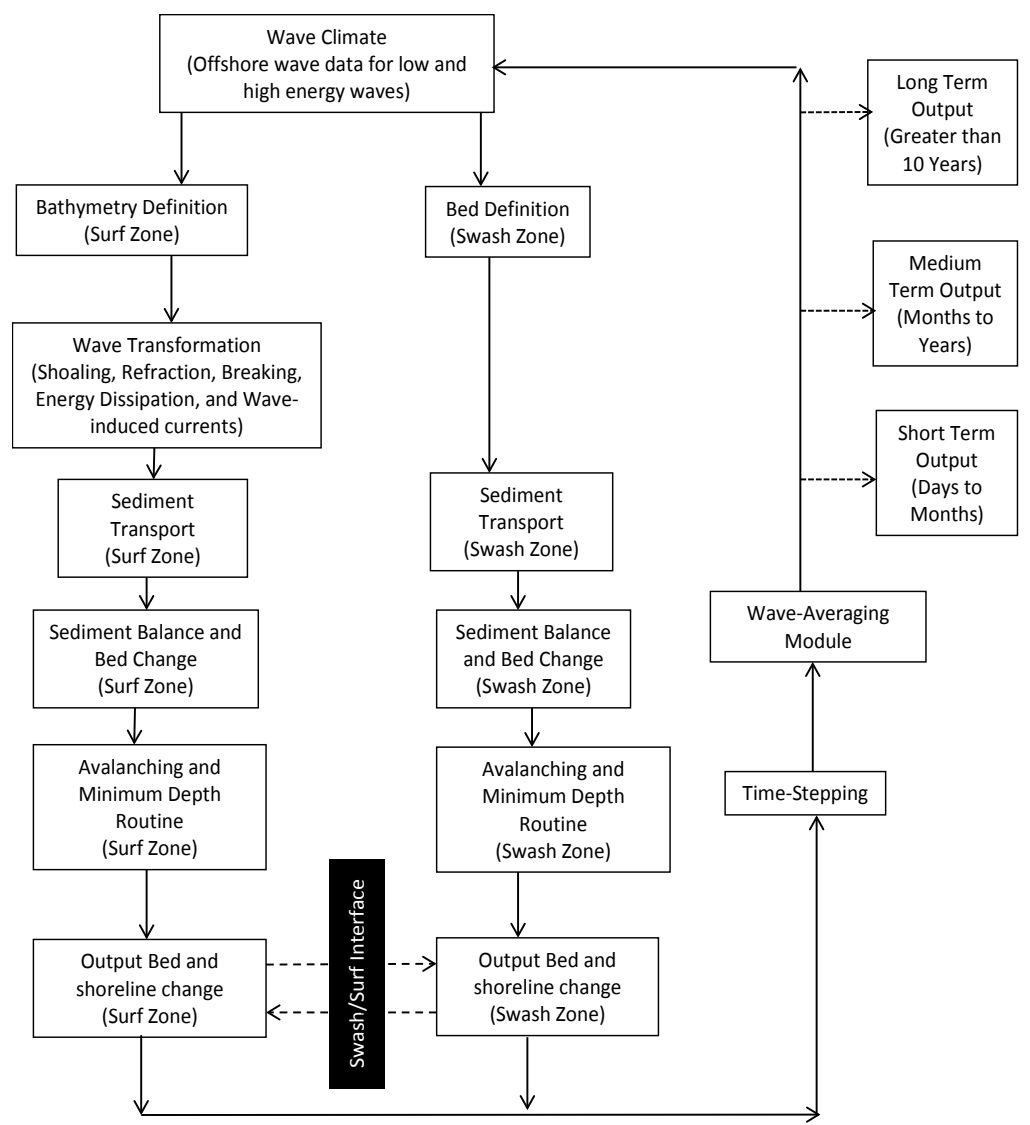

Figure 1. Interaction diagram showing the various modules of the numerical model.

The numerical model includes all the significant natural processes, and in particular storm activity and sea level changes, which are prevalent features on the beaches of the Caribbean (Villarroel-Lamb, 2007b). This numerical model represents a unique effort to quantify morphological response in the Caribbean. It possesses features which distinguish this model from similar models developed elsewhere, such as the simplified representation of the effects of non-linear waves, the use of a new depth-averaged scheme to represent undertow velocities, the coupling of surf zone and swash zone sediment models to predict long-term beach behaviour, the use of long-term wave statistics for a Caribbean coastline, the impacts of storm/hurricane conditions (defined stochastically) on the long-term coastal morphology, the impact of sea level changes through random sampling from a defined distribution and the consideration of sheltering of some Caribbean coasts (Villarroel-Lamb, 2006). The waves are applied at the deep water boundary of the nearshore zone and are transformed from that boundary to the shoreline. The applicable physical processes have been implemented through new mathematical expressions, derived from theoretical analyses and empiricisms, examples of which are given in Villarroel-Lamb (2010) and Villarroel-Lamb (2007a). Figure 2 illustrates the flow chart connecting the various model components.

The model yields beach responses at short-term, medium-term and long-term timescales, for high wave energy and relatively lower wave energy periods. The long-term morphological responses are predicted by integrating the effects of the short-term processes within a probabilistic framework. The 
numerical model also uses a wave-averaging feature which was developed to reduce simulation run time, but makes assumptions about the wave persistence over the given wave interaction period. Nonetheless, this feature allows the generation of a large number of simulation runs to be more computationally efficient. The numerical model can be used in both a deterministic mode and a probabilistic mode. In the deterministic mode each simulation run, will yield the same result each time the model is run. However, in probabilistic mode, each simulation run will yield a different result each time the model is run.

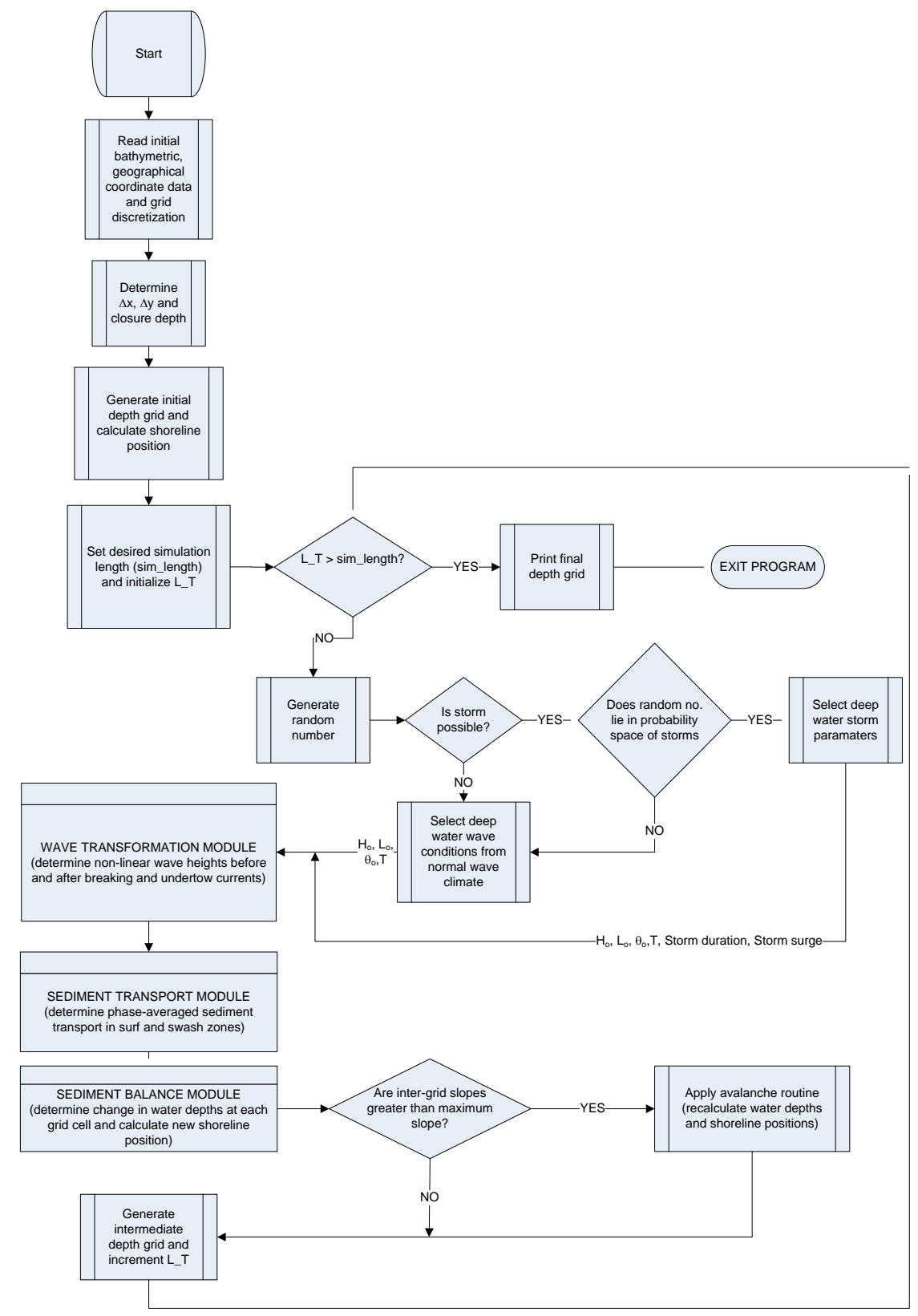

Figure 2. Flow chart illustrating main model components.

The modeling is continually undergoing further development to enhance its features. There are those improvements which are immediately noticeable, such as the implementation of a graphical user interface (GUI) to improve user-friendliness. The GUI is designed to be simple and easy to use and a screenshot is shown in Figure 3. 


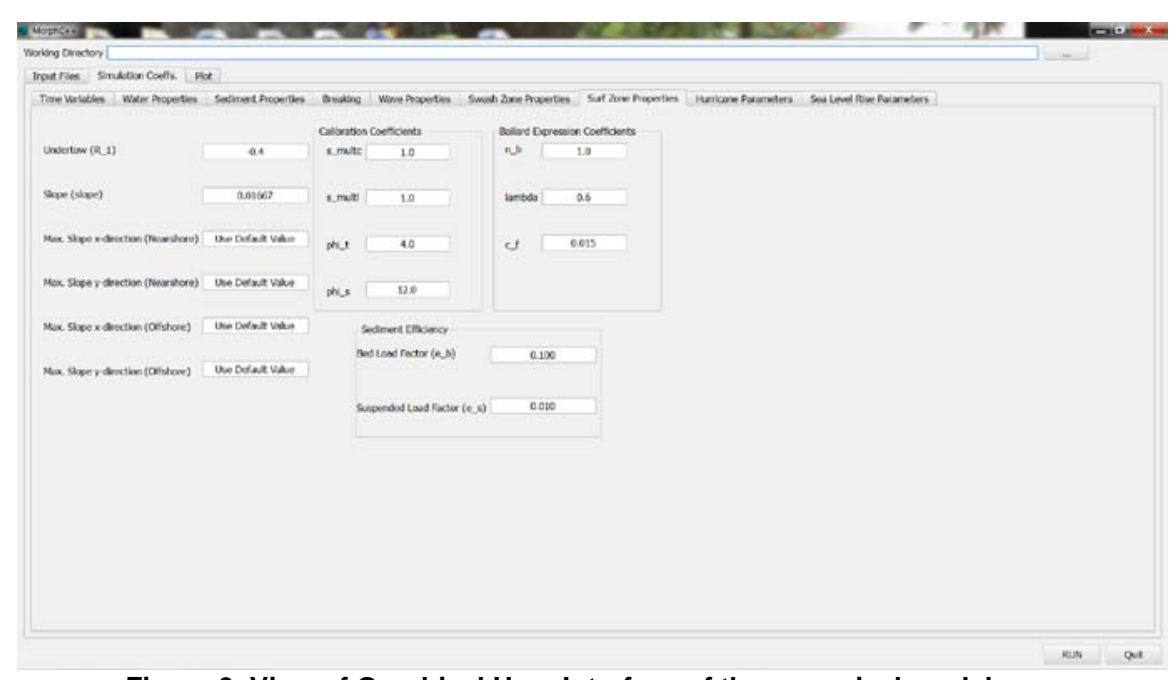

Figure 3. View of Graphical User Interface of the numerical model.

However, considerable effort has been placed on those activities that are not immediately visible by the user. One such activity is the re-writing of the code from procedural $\mathrm{C}$ to employing the Object Oriented Programming capabilities of $\mathrm{C}++$. There has been significant effort on the development of the swash zone model, which now can describe changes occurring in the sub-aerial zone of the beach and not only the sub-aqueous areas of the coastal zone. The parametric formulations are always being updated as the results from additional testing yields useful insights. One such example is the enhancement of the wave runup feature which now incorporates the ability to facilitate changes in runup height due to infiltration and exfiltration processes on the beach. More often than not, each enhancement or detailing of a given coastal process has resulted in the increase in model runtime. Therefore, in conjunction with the other improvements, various techniques had to be investigated to reduce the model runtime.

There are various avenues being explored to increase computational efficiency. These options may be categorized as (a) changes in the overall programming structure (b) improvements in computing hardware and (c) changes in the numerical techniques used in the model. Parallel programming is the main option being explored under changes in the overall programming structure, but the investigation is only in its preliminary stages and has so far not produced significant change in runtime. In order to improve the computing hardware, options that use CUDA programming are also being explored. CUDA is a parallel computing construction developed by NVIDIA and available using a CUDA-enabled graphics processing unit (GPU). It affords the user enhanced computational efficiency by using the powerful capabilities of the GPU (Sanders and Kandrot, 2011). This is a key element to consider so that there is no requirement to have a computing cluster to access the increased efficiency that parallel programming algorithms will afford. The program may be run on a stand-alone computer even if the parallel computation of model functions is achieved. However, changes to the numerical techniques used in the model have been the dominant focus thus far. One option that has been brought to fruition is the enhancement of the wave-averaging feature of the model.

If unadjusted, the model requires significant computational effort to estimate short to medium term shoreline change for a series of randomly selected waves. Long-term changes require large amounts of computing time, and it is necessary to increase the computing efficiency of the model through use this wave-averaging mechanism. This feature of the model allows a shorter computational run for the same assigned morphological time period to be simulated. As such, it provides a means to run simulation periods of longer duration, such as long-term changes.

The wave-averaging technique comprises sampling from the same assigned probability distributions from which the probabilistic wave climate is sampled. However, this feature makes the assumption that the selected wave provides the hydrodynamic forcing over a period of time greater than its wave period, the 'wave interaction period'. The user defines this 'wave interaction' period, over which the bathymetry and the incident offshore wave are assumed to remain unchanged. The bathymetry is updated at the end of the wave interaction period. In other words, the morphological change is averaged over the assigned wave interaction period, based on the beach response to the selected offshore wave. 


\section{METHODOLOGY}

This section describes, in detail, how the response parameter is assessed and analyzed. Through the use of a case study the beach response is investigated by comparing certain key parameters. Firstly, a site was selected for which beach profile data was readily available. This site was located within the Cocos Bay, which is situated on the east coast of Trinidad. Trinidad is one of the islands of the twinisland republic, Trinidad and Tobago. It is the most southerly island of the Caribbean adjacent to the South-American continent. Figure 4 shows the location of the study site, Cocos Bay. Cocos Bay is bounded by two headlands, Manzanilla Point and Radix Point and the study site, a 5km length of coastline, is located just north of the midpoint of the bay. Cocos Bay is generally mild, having an average nearshore slope of $1 / 300$ with steeper bed slopes further offshore.

This investigation considers an overall assessment of the predictive capability of the numerical model when it used in both a deterministic mode and a probabilistic mode.

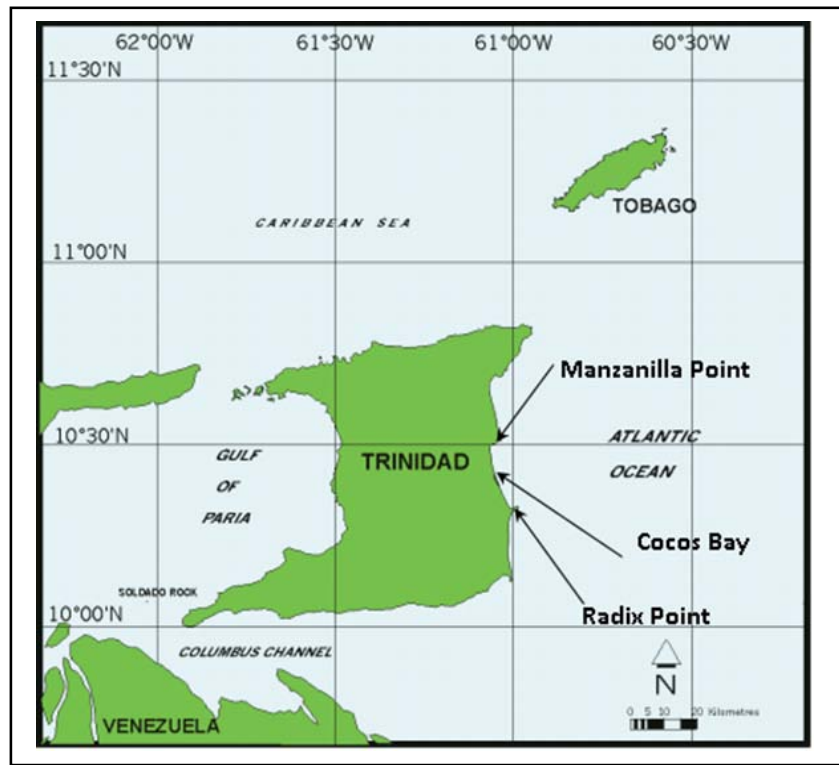

Figure 4. Location map of the study site (from www.ima.gov.tt/CEPintro.html).

The first aspect of this investigation involves the comparison of similar modules between the numerical model (using the non-linear wave option) and the commonly used coastal modeling software, DHI's MIKE 21. The model comparison focuses on the wave height predictions in the nearshore zone, the near bed velocities and the local sediment transport. The SW module of the MIKE 21 suite was used for the comparison on the wave heights and near bed velocities in the nearshore zone, while the ST module of the MIKE 21 suite was used for the comparison of the local sediment transport under a given wave condition. Only the effect of waves is investigated in these comparisons and no other currents are incorporated in the model simulations. For this comparison the numerical model is run in deterministic mode. It is also important to note that the model comparison to MIKE 21 compares order of magnitudes and general trends. It assesses whether the output can fall within the same range when compared to the commonly applied software suite, MIKE 21. Therefore, a simple model case was chosen where the significant offshore wave height, $\mathrm{H}_{\mathrm{so}}$, is equal to $5 \mathrm{~m}$; the peak wave period, $\mathrm{T}_{\mathrm{p}}$, is equal to $10 \mathrm{~s}$; and where waves are approximately normally incident to the shoreline. Two values of the breaker index, $\gamma$, were used in MIKE $21 \mathrm{SW}$ and these were values of 0.78 and 1.1. The median sediment grain diameter used in both models was $0.25 \mathrm{~mm}$, with a bed porosity of 0.4 .

The second aspect of the response parameter analysis is the investigation of the probability distribution of the randomly generated shoreline positions. For these analyses the new numerical model is run in probabilistic mode and a number of simulations of the response parameter, that is the shoreline position, were completed using the model for the case study identified. The shoreline location is defined by a series of points which are given as distances from some reference point. In these analyses, eleven points were used to define the shoreline and their distances from the deep water limit are used to indicate the shoreline location. Shoreline location point 1 is the most northerly point of the study site, shoreline location point 11 is the most southerly point of the study site, with shoreline location points 
evenly spaced within the $5 \mathrm{~km}$ stretch of coastline. One set of shoreline points at the end of a single simulation run define the shoreline location for that simulation run.

The numerical model was run one hundred and fifty times to yield the results used in this analysis. In the first instance, the SPSS Statistics software package was used for this investigation to analyze the best probability distributions. In addition, MATLAB was used to provide any additional data for the analyses, such as more detailed figures or charts. The 'dfittool' feature in MATLAB allows a number of probability distributions to be investigated for best fit on the data, using a visual comparison. Graphs were generated and the probability distributions that best fit the generated shoreline locations were highlighted. This investigation of the best fit probability distribution not only involved the determination of whether the predicted beach responses follow a defined distribution, but also the determination of whether that distribution changes with the number of simulated results and whether any convergence of the distribution will occur as the number of simulation runs increase. The main purpose of this investigation is to conclude whether the numerical tool can yield results which are suitable for application to a quantitative risk assessment of the coastal erosion hazard on a given coastline.

The final aspect of the response parameter analysis is the investigation of the effect of the waveaveraging feature on the output result. The numerical model was run in deterministic mode for this aspect of the investigation which assesses the shoreline positions given varying wave interaction periods. The wave interaction period is the duration that a selected wave is assumed to persist and is usually given as a product of the wave period of the selected wave, for example 1 wave period, 10 wave periods or 100 wave periods. This procedure is equivalent to randomly selecting a smaller number of waves to represent the wave climate over the same simulation period. The shoreline position is determined for wave interaction periods of $1,5,10,25,50,75,100,250,500$ and 1000 wave periods. The relative difference is determined for each wave interaction period where a wave interaction period of 1 wave period is used as the reference.

\section{RESULTS}

The results will be presented in three separate sections to emulate the three separate types of investigative analyses performed for this paper: the MIKE 21 comparison, the generated shoreline statistics and the wave-averaging investigation.

\section{MIKE 21 Comparison}

The first set of results presented in this section is the wave height comparison. Figure 5 shows the comparison of the wave heights where the change is greatest, i.e. near the shore. Figure 6 shows the absolute differences and relative differences respectively, between the results of the new numerical model and those of the MIKE 21 runs.

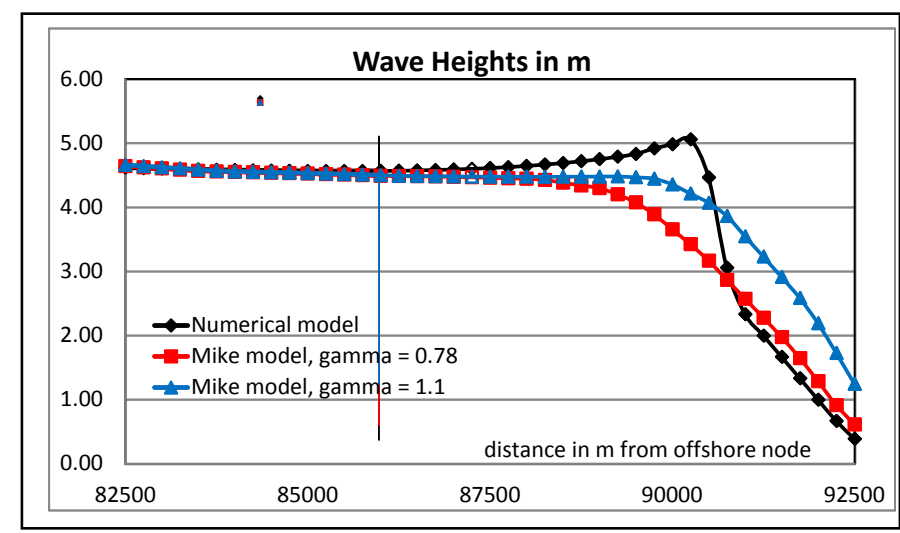

Figure 5. Comparison of the wave heights generated in MIKE 21 and the numerical model. 


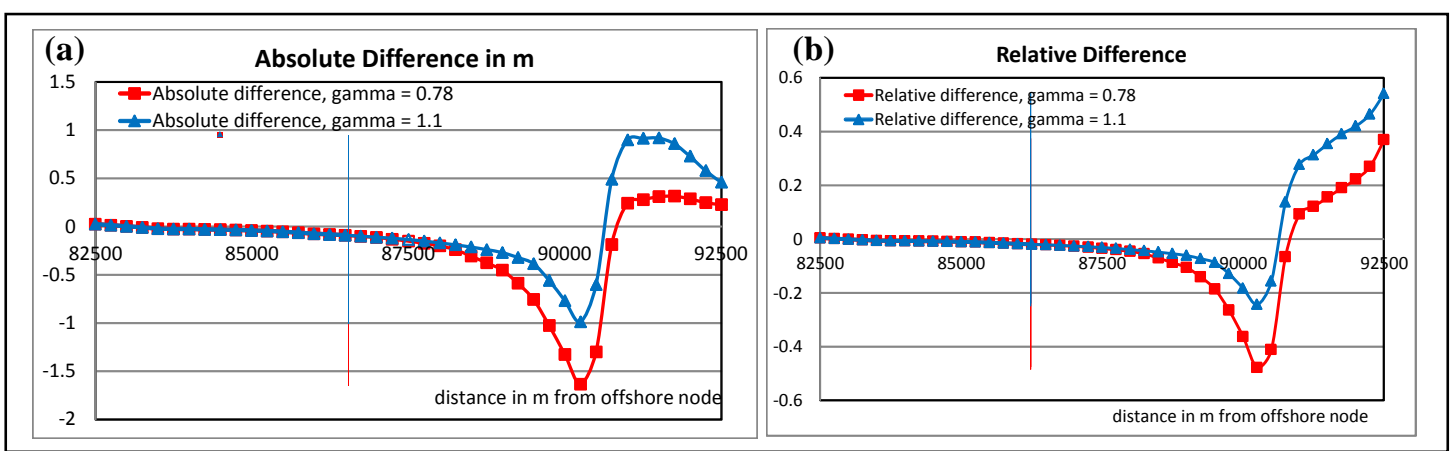

Figure 6. (a) Absolute and (b) Relative difference between the wave heights generated in MIKE 21 and the numerical model.

The second set of results shown in this section is the comparison of the wave generated nearbed velocities. Figure 7 shows the comparison of the nearbed velocities near the shore. Figure 8 shows the absolute differences and relative differences respectively, between the nearbed velocities of the new numerical model and those of the MIKE 21 runs.

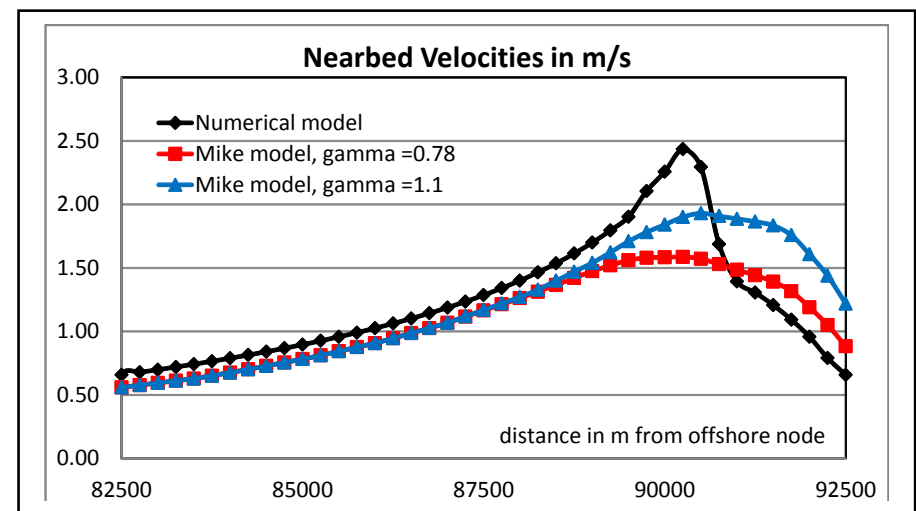

Figure 7. Comparison of the nearbed velocities generated in MIKE 21 and the numerical model.

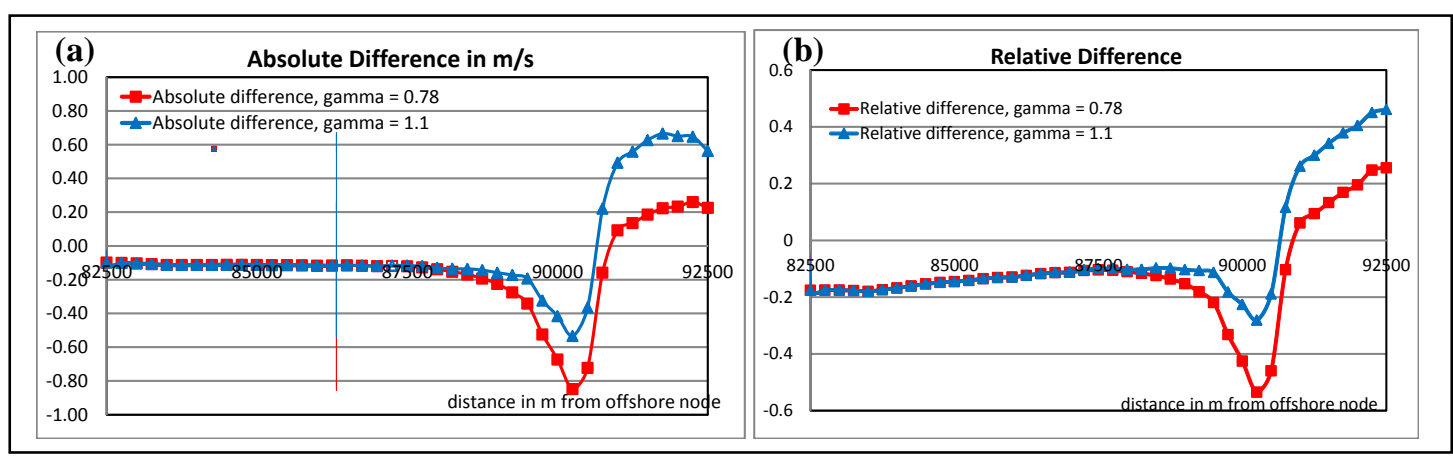

Figure 8. (a) Absolute and (b) Relative difference between the nearbed velocities generated in MIKE 21 and the numerical model.

The last set of results shown in this section is the comparison of the sediment transport rates under the action of the wave. Figure 9 shows the comparison of the sediment transport rates near the shore. Movement of sediment in the cross-shore direction is given by the sediment rates in the x-direction and the transport in the longshore direction is given by sediment rates in the y-direction. Figure 10 shows the absolute differences and relative differences respectively, between the sediment transport rates of the new numerical model and those of the MIKE 21 runs. 


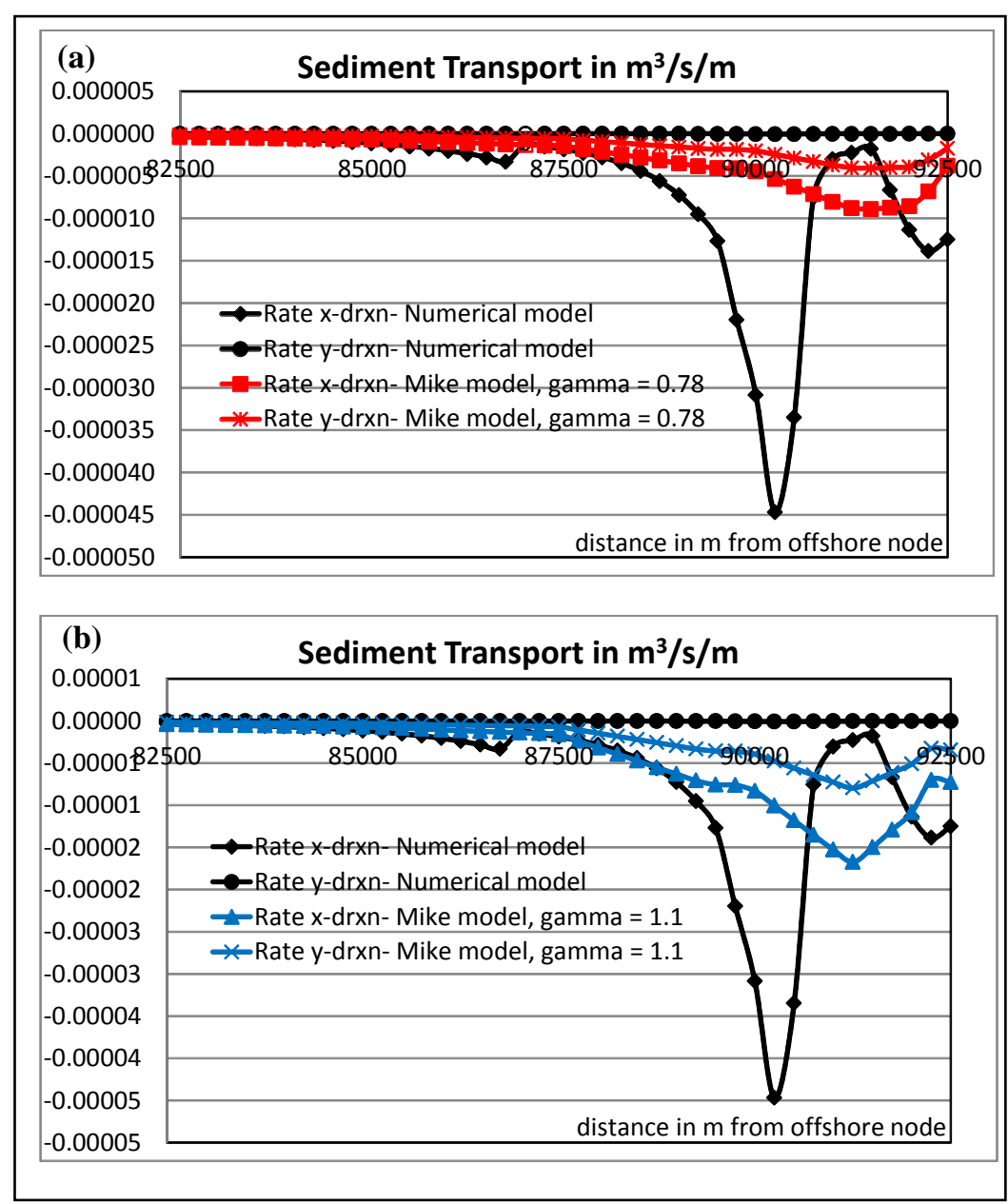

Figure 9. Comparison of the sediment transport rates generated in MIKE 21 and the numerical model for (a) the $x$-direction and (b) the $y$-direction.

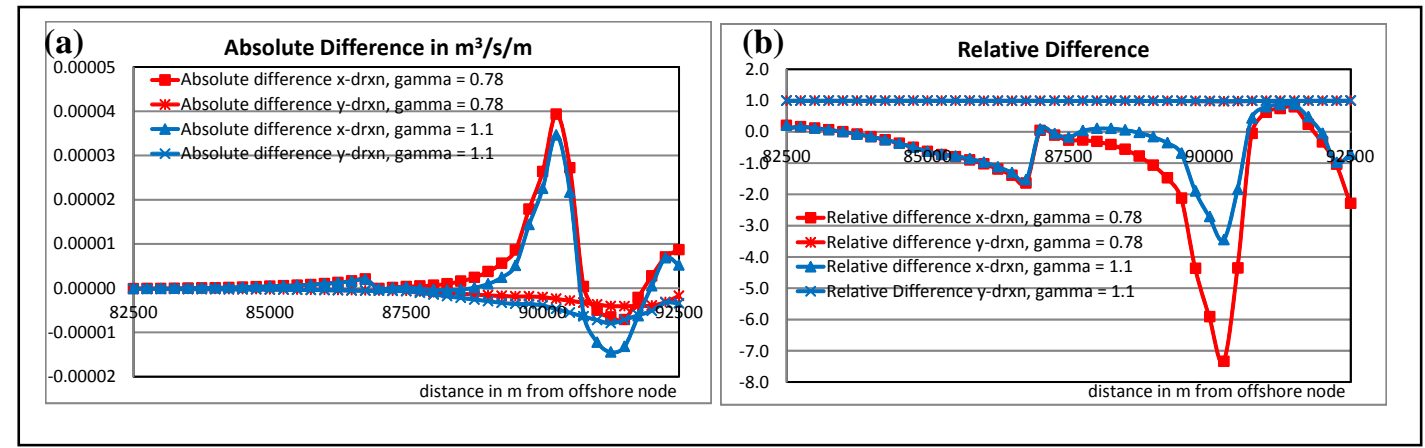

Figure 10. (a) Absolute and (b) relative difference between the sediment transport rates generated in MIKE 21 and the numerical model.

\section{Generated Shoreline Statistics}

The analysis data generated from these tests were quite extensive and the presentation of the results in this section will be limited to representative samples. Only three shoreline location points 1, 6 and 11 , has been illustrated in these results using the SPSS Statistics and MATLAB software packages. Further to which only a subset of the SPSS results for these points will be shown.

The results of the application of the dfittool in MATLAB are shown in Figures 11, 12 and 13 for shore location points 1,6 and 11 respectively. 


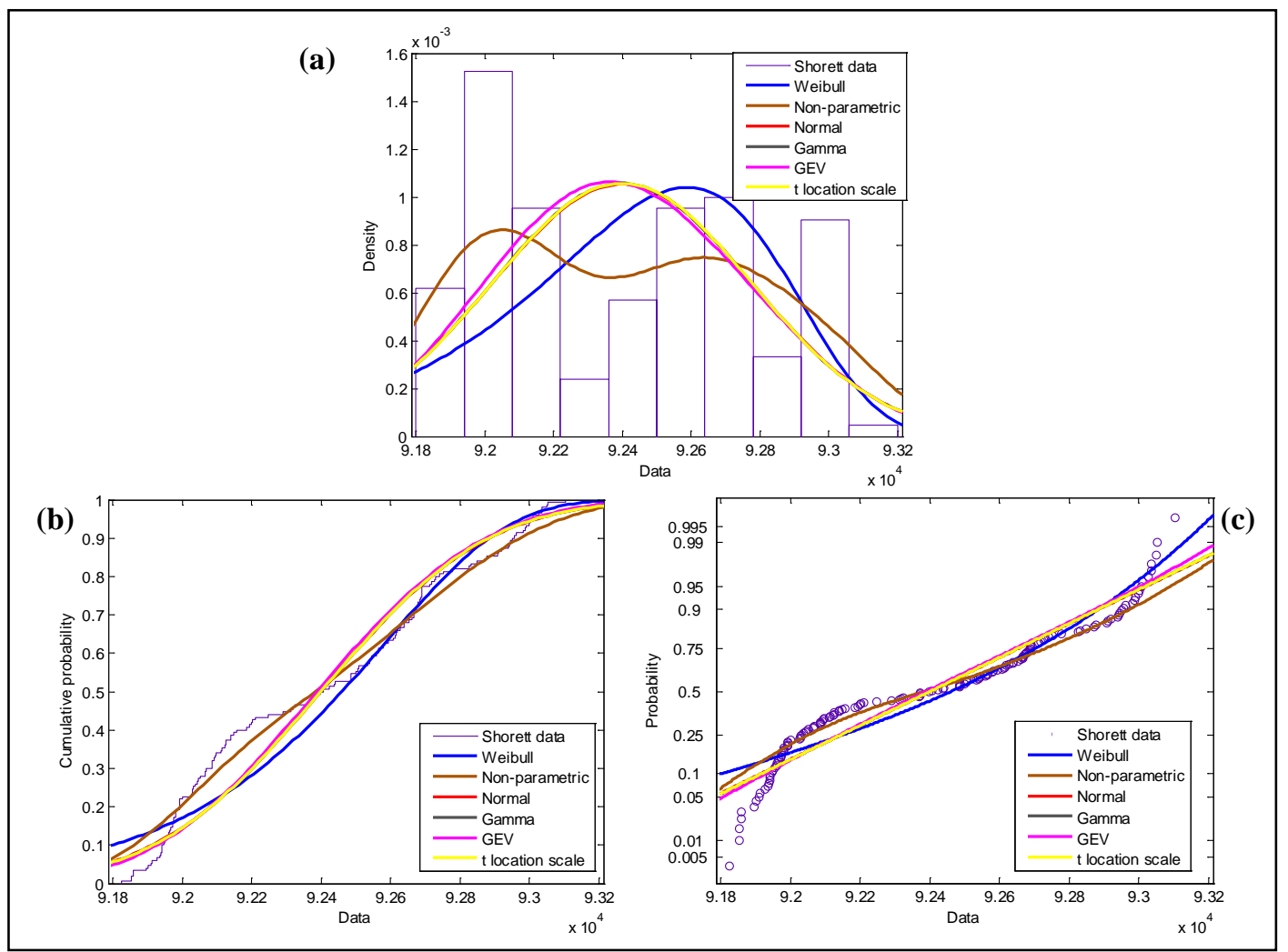

Figure 11. The fitting of various probability distributions at Shore Location Point 1 using MATLAB: (a) Probability density functions, (b) Cumulative distributions and (c) P-P plots.

(a)
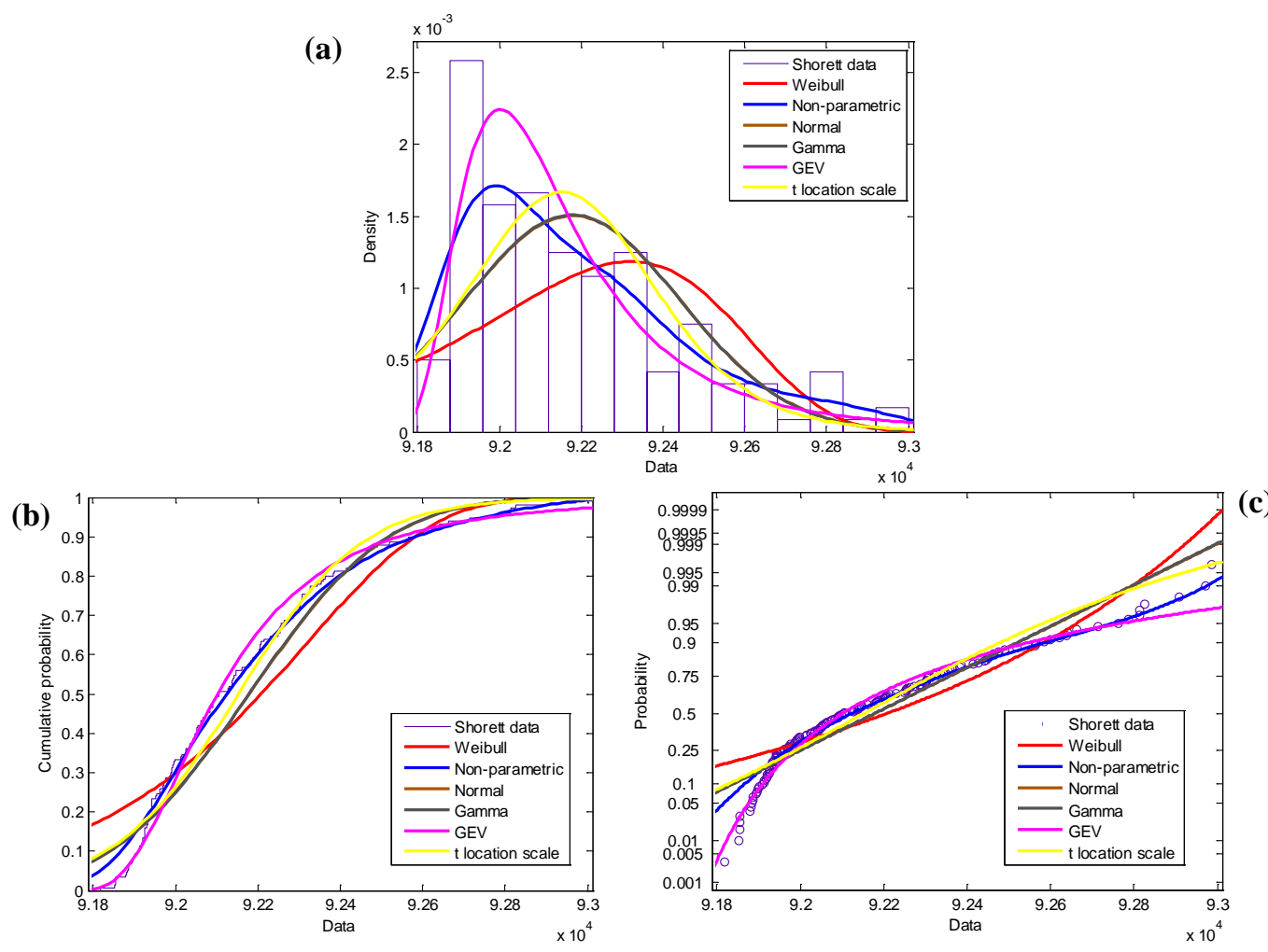

Figure 12. The fitting of various probability distributions at Shore Location Point 6 using MATLAB: (a) Probability density functions, (b) Cumulative distributions and (c) P-P plots. 


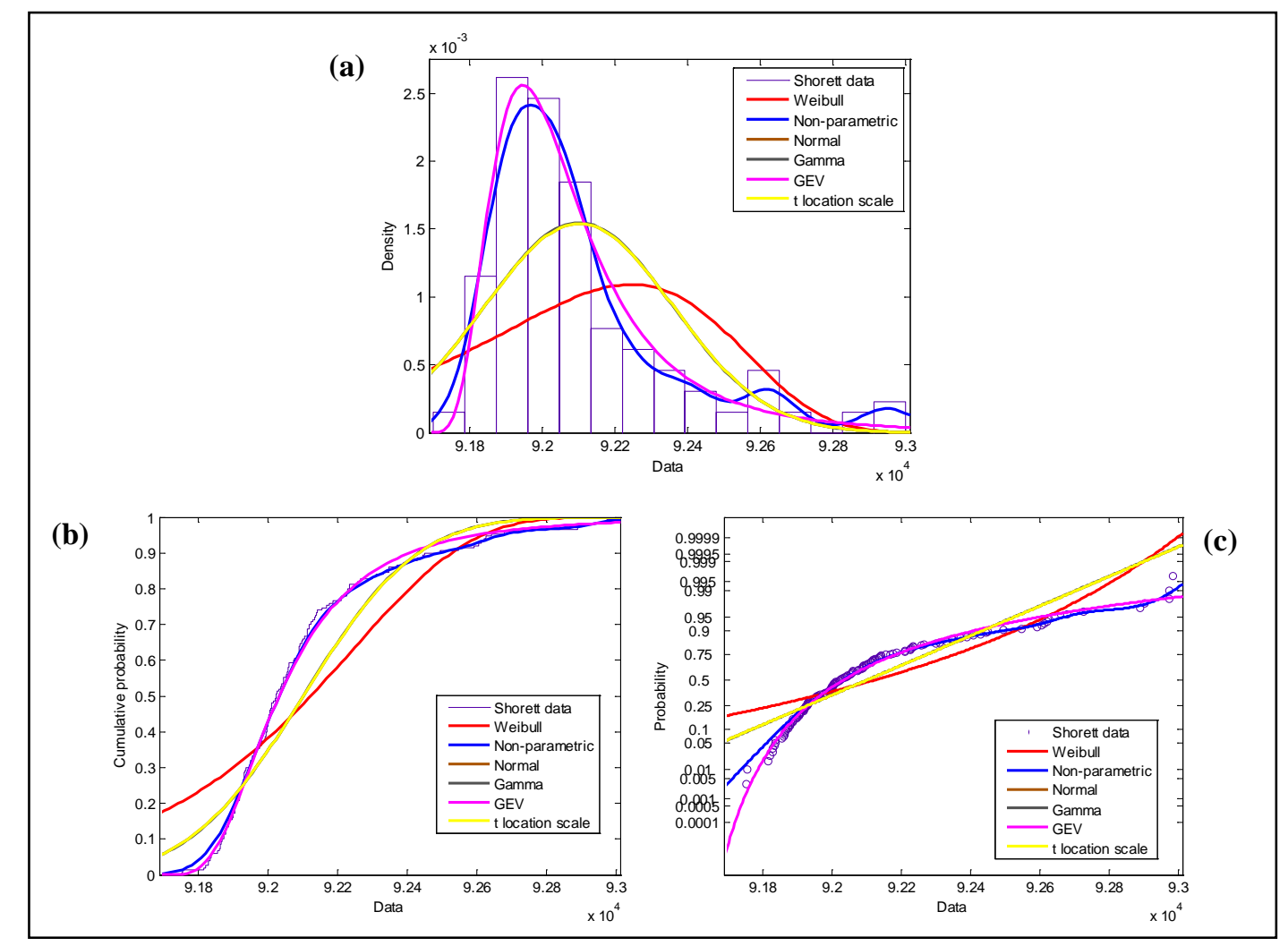

Figure 13. The fitting of various probability distributions at Shore Location Point 11 using MATLAB: (a) Probability density functions, (b) Cumulative distributions and (c) P-P plots.

Some of the results of the analyses using the SPSS Statistics software are shown in Figures 14, 15, 16 and 17 for shore location points 6 and 11 only. Figure 14 shows the P-P plot and detrended P-P plot for the Weibull distribution while Figure 15 shows the P-P plot and detrended P-P plot for the Normal distribution, for shore location point 6.

Figure 16 shows the P-P plot and detrended P-P plot for the Weibull distribution while Figure 17 shows the P-P plot and detrended P-P plot for the Normal distribution, for shore location point 11.

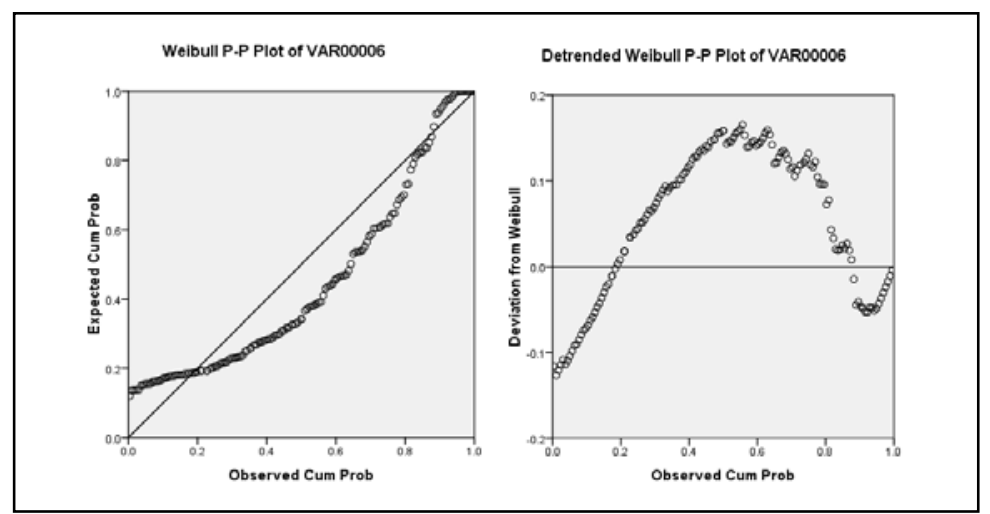

Figure 14. P- P plot and detrended P-P plot for the Weibull distribution for shoreline location point 6. 


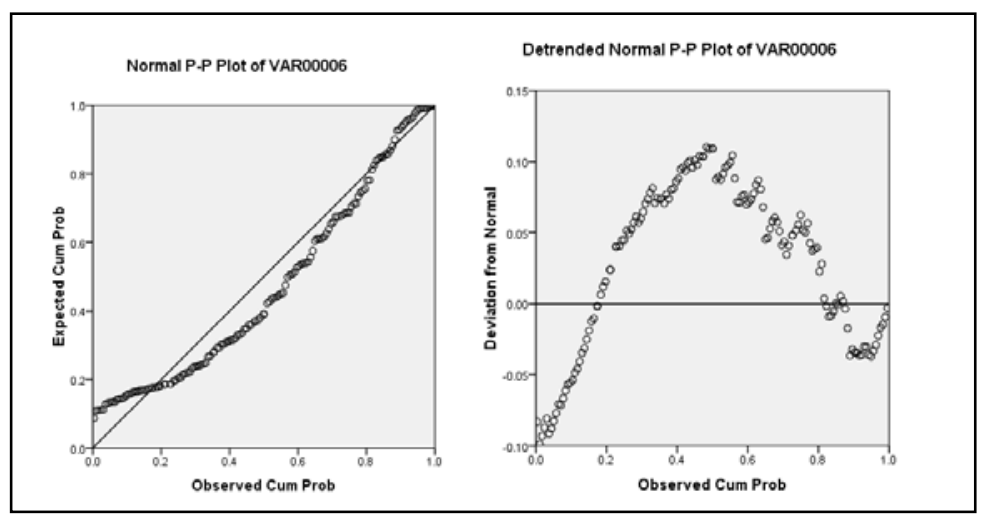

Figure 15. P- P plot and detrended P-P plot for the Normal distribution for shoreline location point 6.

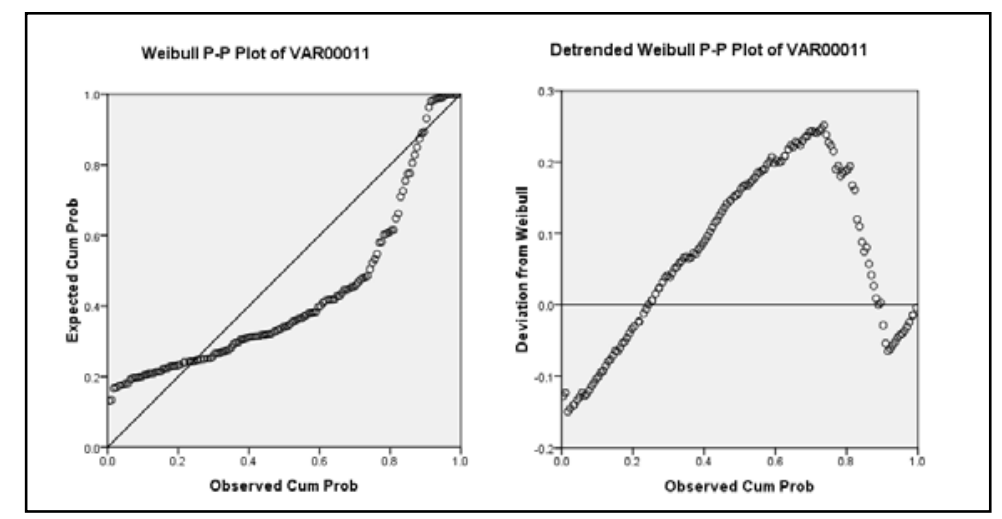

Figure 16. P- P plot and detrended P-P plot for the Weibull distribution for shoreline location point 11.

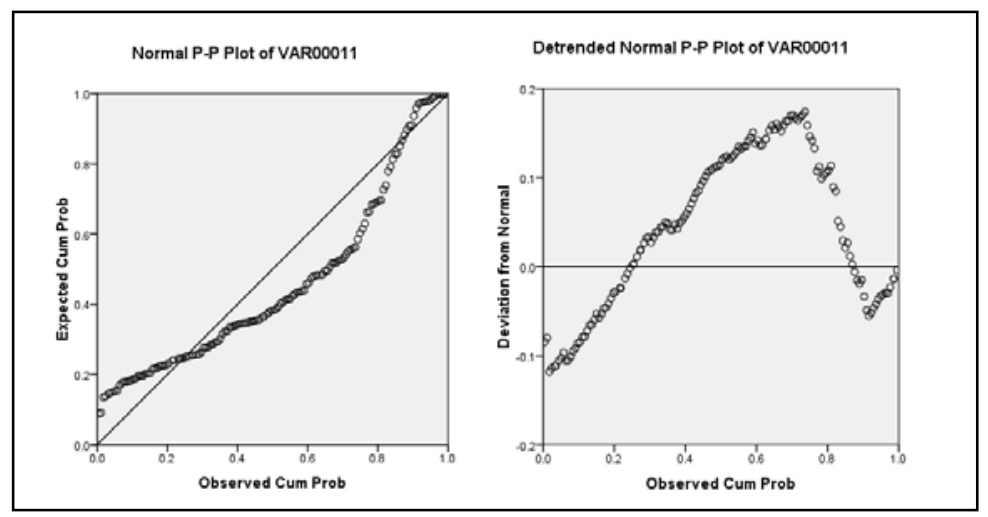

Figure 17. P- P plot and detrended P-P plot for the Normal distribution for shoreline location point 11.

\section{Wave-averaging Investigation}

The results of the wave-averaging investigation are given in Table 1 below and illustrated in Figure 18. Table 1 shows the relative difference (correct to 3 d.p.) of the shoreline position of each shoreline point for the tested wave interaction period (abbreviated as WIP in the Table), referenced to the shoreline position where the wave interaction period is 1 wave. 


\begin{tabular}{|c|c|c|c|c|c|c|c|c|c|c|c|}
\hline $\begin{array}{c}\text { Shoreline } \\
\text { Points }\end{array}$ & 1 & 2 & 3 & 4 & 5 & 6 & 7 & 8 & 9 & 10 & 11 \\
\hline \multicolumn{12}{|l|}{$\begin{array}{l}\text { WIP (no. } \\
\text { of waves) }\end{array}$} \\
\hline 1 & ? & 0.000 & 0.000 & 0.000 & 0.000 & 0.000 & 0.000 & 0.000 & 0.000 & 0.000 & 0.000 \\
\hline 5 & -0.001 & -0.001 & -0.002 & -0.001 & -0.002 & -0.001 & -0.001 & -0.005 & -0.007 & -0.007 & -0.009 \\
\hline 10 & -0.004 & -0.006 & -0.006 & -0.003 & -0.006 & -0.006 & -0.006 & -0.007 & -0.006 & -0.008 & -0.008 \\
\hline 25 & -0.005 & -0.006 & -0.006 & -0.006 & -0.006 & -0.005 & -0.005 & -0.005 & -0.005 & -0.006 & -0.006 \\
\hline 50 & -0.008 & -0.007 & -0.009 & -0.008 & -0.008 & -0.008 & -0.007 & -0.006 & -0.007 & -0.007 & -0.007 \\
\hline 75 & 0.003 & -0.004 & -0.005 & -0.007 & -0.006 & -0.006 & -0.006 & -0.002 & -0.001 & -0.005 & -0.005 \\
\hline 100 & 0.000 & 0.000 & 0.001 & 0.001 & -0.004 & -0.005 & -0.007 & -0.007 & -0.006 & 0.001 & 0.000 \\
\hline 250 & -0.003 & -0.006 & -0.003 & 0.001 & 0.002 & 0.002 & 0.002 & 0.002 & 0.002 & 0.002 & 0.002 \\
\hline 500 & -0.005 & -0.004 & -0.004 & -0.003 & -0.005 & -0.004 & -0.004 & -0.006 & -0.002 & -0.005 & -0.004 \\
\hline 1000 & 0.006 & 0.006 & 0.006 & 0.005 & 0.005 & 0.005 & 0.007 & 0.003 & -0.001 & -0.003 & -0.004 \\
\hline
\end{tabular}

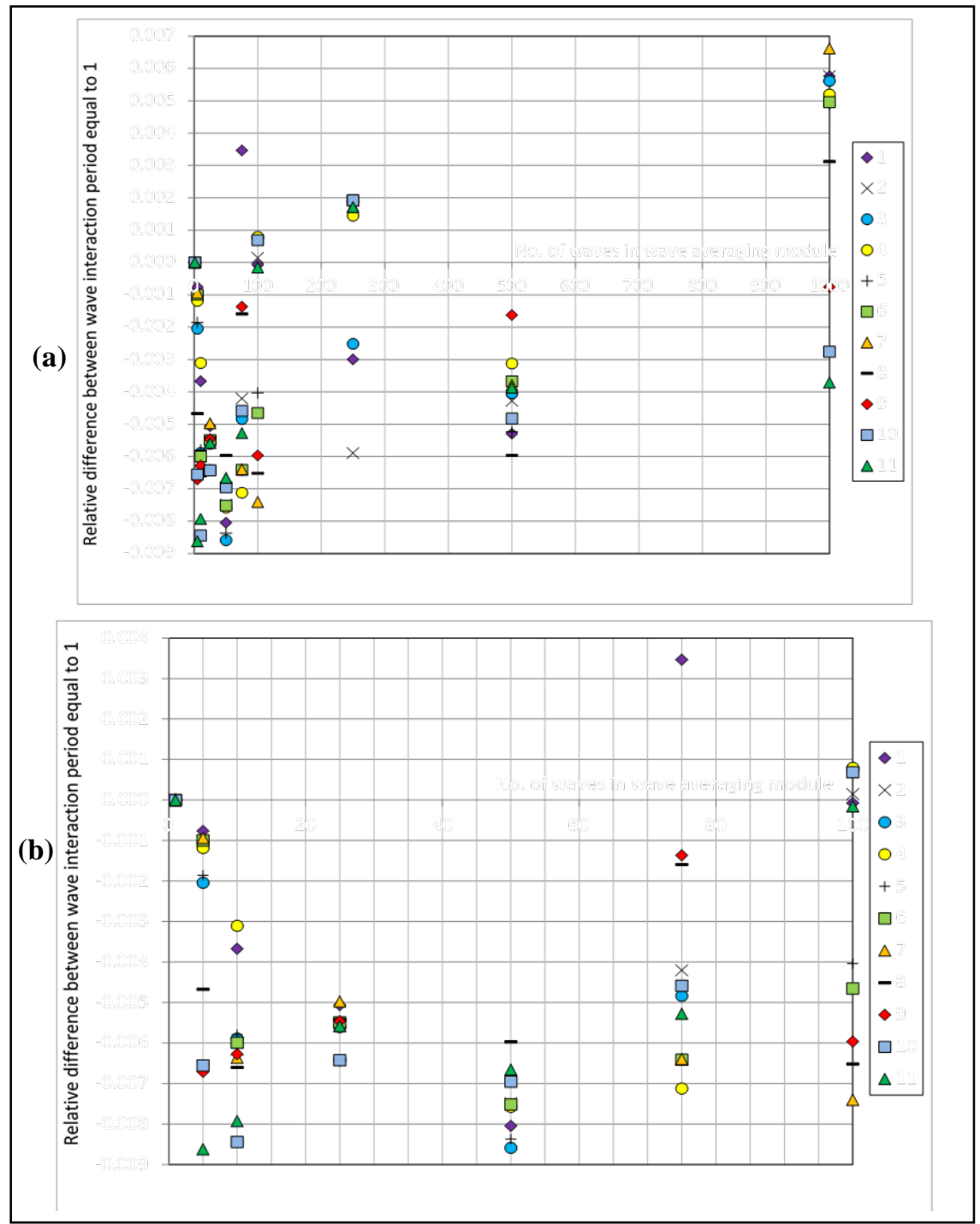

Figure 18. Plot of the relative differences versus wave interaction period for the $\mathbf{1 1}$ shoreline location points: (a) Plot shows all wave interaction periods (b) Plot shows only from 1 to 100 waves for the wave interaction period. 


\section{DISCUSSION OF RESULTS}

\section{MIKE 21 Comparison}

The comparison of the wave heights between the numerical model and the results generated in the SW module were similar until breaking occurred (Figures 5 and 6). The MIKE model did not produce the peak at breaking that was generated in the numerical model. Currently, the numerical model gives the user two options for the determination of incipient breaking, Weggel (1972) and Ostendorf and Madsen (1979) formulations. Both formulations produce the significant peak in wave height at breaking, which is not observed in the MIKE 21 SW model, even when the MIKE model uses a constant breaker index of 1.1. This is the most notable difference in the results generated. The differences in wave height after breaking is not a significant feature since the calibration parameter in the numerical model can be easily adjusted to match a given data set. In the new model, the wave height after breaking is determined using the Battjes and Janssen (1978) formulation for the dissipation model where the breaker parameter in that formulation is represented by the Kuriyama and Ozaki (1996) equation. The calibration coefficient in that equation can be easily adjusted for calibration purposes. The differences at breaking are also clearly evident in the comparison of the nearbed velocities which reflects the differences observed in the wave heights magnitudes (Figures 7 and 8).

The comparison of the sediment transport rates also illustrate the differences in wave heights at breaking, showing higher sediment transport than that of the MIKE ST module, at breaking (Figures 9 and 10). A relative difference of the wave heights, at breaking, of 0.24 (in other words the numerical model produces wave heights 0.24 times higher than the MIKE SW at breaking), produces nearbed velocities that are 0.28 times higher than the MIKE SW at breaking. However, the same wave condition produces sediment transport rates 3.44 times higher than the MIKE ST at breaking. This reflects differences in the sediment transport models used in each of the two numerical models compared (i.e. MIKE 21 and the new model). The new numerical model uses energetics-type, phase-averaged sediment transport expressions to ascertain the expected sediment transport rates. Potential sediment rates are determined, for both the surf and swash zones, using modified Bailard (1981) expressions. Another noteworthy difference is the peak at which the maximum sediment transport rate occurs. The new model shows a maximum sediment rate at breaking point, while the MIKE 21 model shows a maximum sediment rate just landward of the breaking point. These differences will be reflected in the calculation of bed changes and the location of offshore bar and trough systems.

\section{Generated Shoreline Statistics}

The results of this analysis indicate that the response parameter is best described by a single defined probability distribution, the generalized extreme value distribution (Figures 11, 12 and 13). The generalized extreme value (GEV) distribution is a three-parameter model that combines the Type I, II and III maximum extreme value distributions. In all cases examined (where only a representative sample is shown in the Results section of this paper), the GEV distribution generally was the best fit for all data sets. In some cases, as shown in Figure 11, a non-parametric distribution was the best fit as it imitated the peaks in density of the data set. However, the GEV distribution still represented the best fit over all data sets. Figures 14 to 17 are used to highlight the poor fit of other probability distributions (the Weibull and Normal distributions are used as examples in these figures).

In addition, it appears that this type of probability distribution is the best fit for the dataset of results and this is reinforced as this best-fit distribution remains the same even with differences in the number of simulation runs used.

\section{Wave-averaging Investigation}

These results indicate that the wave-averaging feature has a significant impact on the response parameter. Although, some type of effect was expected from the wave-averaging feature, the magnitude of the influence is a noteworthy characteristic of the numerical model.

The analysis assumes that the wave interaction period of 1 wave period is ideal and hence compares all subsequent wave interaction periods to these results. In Table 1 and Figure 18, negative values indicate a shoreline position seaward of the reference shoreline (accretionary trend); while a positive value indicates a shoreline position landward of the reference shoreline (erosionary trend). Figure 18 illustrates that the differences in values, from the reference shoreline, oscillate as the wave interaction period increases, and do not follow a constant trend. Along the shoreline (i.e. for different shoreline location points) for the simulation run, the values of the relative differences vary significantly. For differing shoreline location points, using the same wave interaction period, both positive and negative differences are observed. It is surprising that a wave interaction period of 500 waves produce results 
that are closest in value to the reference wave interaction period and are all negative. It is worthy to note that the relative values shown in Table 1 are small in magnitude, but when applied to distances on the order of tens of kilometers, the differences in shoreline position represent substantial values (which was the case for this particular case study).

The computational efficiency is greatly increased using the wave-averaging module. If a 1 year simulation is run using a wave interaction period of 1 wave, the model takes approximately 5 calendar days to complete the simulation. However, using a wave interaction period of 100 waves the simulation time is reduced to 20 minutes. This affords the user the capability of increasing the number of simulation runs to extract probabilities of shoreline position for further analyses to facilitate coastal planning. Therefore, the model must also be calibrated using the wave-averaging module at varying levels to ensure that the best balance is obtained between accuracy and computational efficiency for the generated results.

\section{CONCLUSION}

There may be some variations in the wave heights, nearbed velocities and sediment transport rates of the new model when compared to the relevant MIKE 21 models. The differences in wave-related parameters between the models are observed at wave breaking; however the numerical model does reflect the significant wave shoaling that may occur near the breaking point. Nonetheless, the new model (just like all other models) may be calibrated and/or validated with laboratory or field data to improve predictive capability.

The probabilistic results of the beach shoreline response illustrates that a single probability density function can be generally applied to the shoreline position. The Generalized Extreme Value (GEV) distribution produced the best-fit distribution for most of the data with a non-parametric distribution being a slightly better fit at certain shoreline locations. The probabilistic results of the beach response can therefore be used in a quantitative risk assessment of coastal erosion on Caribbean beaches, but application of the GEV distribution must be subject to prior validation.

The wave-averaging feature is an effective means to reduce the runtime of the numerical model. Use of a given wave interaction period must be validated against the data to ensure that accuracy is not substantially compromised for the sake of computational efficiency. However, once some model validation is done, this feature can improve analyses by allowing a faster run time to facilitate a greater number of runs.

Overall, the model can be used as a predictive tool in coastal planning and management on Caribbean beaches. It has been demonstrated that it can produce useful results and at the very least can be applied in a preliminary assessment of regional beaches.

\section{ACKNOWLEDGMENTS}

The author wishes to acknowledge the support of the Royal Society through granting the author a Newton International Fellowship to advance this research at University College London.

\section{REFERENCES}

Bailard, J. A. 1981. An Energetics total load sediment transport model for a plane sloping beach, Journal of Geophysical Research, 86 (C11), 10938-10954.

Battjes, J. A., and J. P. F. M. Janssen. 1978. Energy loss and set-up due to breaking of random waves, Proceedings of the 16th International Conference of Coastal Engineering, ASCE, 569-587.

Kuriyama, Y., and Y. Ozaki. 1996. Wave height and fraction of breaking waves on a bar-trough beachfield measurements at HORS and modeling, Report of the Port and Harbour Research Institute, 35 (1), 1-38.

Ostendorf, D.W., and O.S. Madsen. 1979. An analysis of longshore current and associated sediment transport in the surf zone, Technical Report Ralph M. Parsons Laboratory for Water Resources and Hydrodynamics, Massachusetts Institute of Technology, Report No. 241, 84-96.

Sanders, J. and E. Kandrot. 2011. CUDA by Example: An Introduction to General-Purpose GPU Programming, Addison-Wesley, Boston, 290 pp.

Villarroel-Lamb, D. A. 2006. Towards the effective prediction of long-term beach morphology on Caribbean coastlines, Fourth LACCEI International Latin American and Caribbean Conference for Engineering and Technology, Mayagüez, Puerto Rico. 
Villarroel-Lamb, D. A. 2007a. Assessing the hurricane-related coastal erosion hazard, Fifth LACCEI International Latin American and Caribbean Conference for Engineering and Technology, Tampico, México.

Villarroel-Lamb, D. A. 2007b. A numerical model to predict long-term coastal morphological behaviour in the Caribbean, PhD Thesis, University of the West Indies, Trinidad and Tobago.

Villarroel-Lamb, D. A. 2010. A phase-averaged non-linear wave transformation model, Proceedings of the Institution of Civil Engineers: Maritime Engineering, 163(2), pp. 73 - 88.

Weggel, J. R. 1972. Maximum breaker height, Journal of the Waterways, Harbors and Coastal Engineering Division, 98 (WW4), 529-548. 